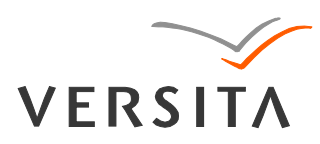

GEOCHRONOMETRIA 40(2) 2013: 90-100

DOI 10.2478/s13386-013-0105-x

Available online at

www.springerlink.com

\title{
A COARSE BAYESIAN APPROACH TO EVALUATE LUMINESCENCE AGES
}

\author{
ANTOINE ZINK \\ Centre de Recherche et de Restauration des Musées de France, Ministère de la Culture, CNRS, Palais du Louvre, Porte des lions \\ 14 quai F. Mitterrand, 75001 Paris, France
}

Received 31 May $2010 \quad$ Accepted 6 February 2013

\begin{abstract}
This paper develops a simplified Bayesian approach to evaluate a luminescence age. We limit our purpose to the cause-effect relationship between the age and the accumulated dose. The accumulated dose is given as a function of the age and several others parameters: internal radionuclides contents, gamma dose rate, cosmic dose rate, alpha efficiency, wetness, conversion factors, wetness coefficients, fading rate and storage time. The age is the quantity we are looking for. Bayes' theorem expresses the changes on the probability distribution of age due to the luminescence study. The information before study (prior) comprises what is previously known about the age and the archaeological model (cultural period, stratigraphic relations, type, etc.) as well as the parameters of the physical model. The accumulated dose consists in the data describing the measurement.

The various stages of Bayesian approach were implemented using the software WinBugs. Simulated data sets were used in various models. We present various small models representing typical examples encountered in luminescence dating.
\end{abstract}

Keywords: Luminescence dating, Bayesian statistics, age equation.

\section{INTRODUCTION}

Unlike other dating methods (Buck and Millard, 2004), luminescence dating methods (thermoluminescence - TL and optically stimulated luminescence - OSL) rarely use Bayesian approach. The literature is limited to a dozen articles. Most (e.g. Rhodes et al., 2003, Clark-Balzan et al., 2012) combine luminescence ages with external information, such as stratigraphic relationships. On the another hand, Sivia et al., (2004) focuses on a very specific step: the assessment of the distribution of equivalent doses in sediment mixtures. Only Huntriss (2008) covers all steps from data acquisition until the final age. We explain this weak attention by the complexity of the principle of luminescence dating.

Corresponding author: A. Zink

e-mail: antoine.zink@culture.gouv.fr
Luminescence dating is based on the ratio between the accumulated dose and the annual dose rate. It thus acts with the physical meaning of a method of absolute dating, which contains in itself its own calibration (Zink, 2009). On the other hand, the large number of parameters needed to determine the age does not encourage users to explore in detail the many sources of uncertainty (Rhodes et al., 2003, Huntriss, 2008). Practitioners of luminescence dating do not feel the need to develop specialized skills in statistics and error treatment. We think for our part, that the Bayesian approach that takes into account all available information, not only measurements of dating, is a plus for our methods.

Huntriss (2008) deals with the determination of the dose equivalent from the results of single aliquot regeneration of optically stimulated luminescence (OSL-SAR) 
measured at different preheat temperatures, as well as the determination of the annual dose in the case of coarse grains using the beta, gamma and cosmic dose rates. The age calculation uses the ratio between the both normal distributions corresponding to the so modelled accumulated and annual dose. In case of combining ages, this model, like the model of Rhodes et al. (2003), does not take into account the presence of uncertainty contributions shared by several age samples.

Our approach is similar to that followed by Millard (2004, 2006a, 2006b). As with other models, it is limited to a stage of the whole luminescence dating: the problem of the assessment of the age from the equivalent dose by means of the age equation. In this limited frame, the aim is to describe, in the best possible way, the whole measurement in order to identify all the sources contributing to age uncertainty. For this purpose, we use the relation of cause and effect (Sommer and Siebert, 2006), as well as the hierarchy of parameters (Lanos, 2004; Millard, 2004, 2006a; Zink, 2009).

We did not try to perform a rigorous model like a statistician, but rather a 'pragmatic' model more in our skills of a practitioner using dating by luminescence. It is therefore not a comprehensive approach that would determine the age from all measures, especially those of luminescence.

\section{MODELLING}

\section{Luminescence dating}

A large number of papers and handbooks provide a clear understanding of luminescence dating (cf. Aitken, 1985, 1998; Preusser et al., 2008; Wintle, 2008; Zink, 2009). Here we give a brief presentation.

To obtain a date, it is necessary have a physical clock. It is characterized by two properties: it must be a physical phenomenon that changes over time; and with, at least, a noteworthy instant.

Luminescence dating is based on the property of minerals to accumulate the energy resulting from the ambient radioactivity. During heating or optical bleaching, the previously stored energy is released as luminescence. We thus have a physical clock to date ceramics and terracotta: the noteworthy event is the initial resetting (last heating or bleaching) and the change over time is a monotonic increase, with a trend mainly linear, for ages below $10 \mathrm{ka}$. To move from this physical clock to a calendar clock, we have to take into account the dose accumulated over one year, by assuming this one constant in time. The ratio of the total accumulated dose to the annual dose yields the age. The total accumulated dose being the physical clock and the annual dose, the calibration due to the environment (radiation, humidity).

It is obviously a brief presentation. Several parameters can introduce errors to the age determination. Among them, in particular, the anomalous fading (Wintle, 1973) should be mentioned, because this phenomenon is com- mon and a mathematical correction at low doses has been proposed (Huntley and Lamothe, 2001).

\section{Cause and effect of a measurement - the age equation}

The overview of luminescence dating given above may result in different protocols, depending on the type of the investigated objects (terracotta, sediments etc.), the analyzed samples (polymineral fine-grains, quartz or feldspar coarse grains etc.), and the available equipment (e.g. TL or OSL reader, dosimeter, spectrometer, alpha counting).

Here we describe the protocol generally followed in our laboratory. It aims to study ceramics; it uses the finegrain technique with additive TL (Zimmerman, 1971) or single aliquot regeneration OSL (Murray and Wintle, 2003, adapted in Zink and Porto, 2005) on samples of small sizes (Zink and Porto, 2005). For the annual dose, we measure the radionuclide contents in the clay itself; the gamma dose is either measured directly or arbitrarily fixed, according to the geological origin of the sample (Zink et al., 2012). Finally, the fading is evaluated by a SAR method (Zink, 2008).

Fig. 1 depicts a cause-effect relationship (Sommer and Siebert, 2006) between the age and the luminescence. To simplify, we split the relationship in two parts, from the age to the accumulated dose and from the accumulated dose to the luminescence. The second part corresponds to a calibration problem (Huntriss, 2008) and will not be considered in this paper. We limit our purpose to the first part: the relationship between the measurand (i.e. the age), the indication (i.e. the accumulated dose) and the influence quantities: radionuclide contents of the clay, conversion factors, alpha efficiency, wetness, parameters of wetness, gamma dose-rate, cosmic dose-rate and fading. All influence quantities, except the fading, contribute to the annual dose.
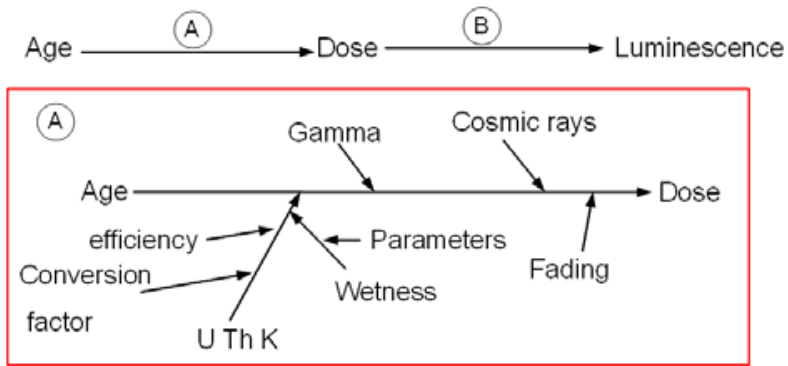

(B)

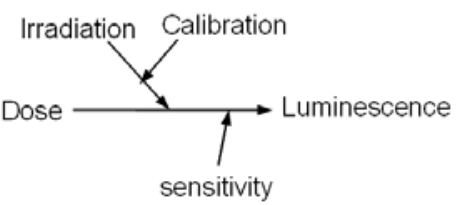

Fig. 1. Cause-effect relationship graph of luminescence dating. 
The age can be represented mathematically by the functional dependence of the accumulated dose on the age and other quantities (Sommer and Siebert, 2006)

accumulated dose $=$ Age $\times$ annual dose $\times$ fading

The inverse equation (model equation) is the equation of age (Aitken, 1985), giving the age according to the accumulated dose

Age $=($ accumulated dose $/$ annual dose $) /$ fading

We note the presence of the correction factor due to anomalous fading. It complicates the equation since this factor depends on the age (Huntley and Lamothe, 2001; Zink, 2008). This should lead us to calculate the age by iteration but it is observed that the number of iterations, even when the fading correction is not negligible, rarely exceeds one or two (Huntley and Lamothe, 2001).

\section{Bayesian approach}

To state experimental results, two general approaches are possible (Cox, 1946). The conventional approach describes the probability to obtain the observed data as a function of the unknown variable, $\operatorname{Pr}(\{$ data $\} \mid$ variable $)$. The second statement called 'conditional' or 'Bayesian' approach describes the probability of the unknown variable according to our observed data, $\operatorname{Pr}($ variable $\mid\{$ data $\})$.

Although the wording is close, there is no identity between both approaches.

The conventional approach assumes that there is no bias between the measured value and the true value. In particular, in the case of repeated measurements under the same conditions, the most probable observed value ('estimate') is assumed to tend towards the true value (Steel, 2001). It is suitable to predict the next draw in a dice roll, or production in a factory (Buck et al., 1996). However in the case of dating, the interest is to determine the most likely age knowing the measured data and then the second approach is best suited. The transition from the classic approach to the Bayesian approach is made through the theorem of Bayes, which describes the probability of $\Theta$, the unknown variable under investigation, as a function of the data and the previous information on the probability of $\Theta$ in the following way (Buck et al., 1996; Buck and Millard, 2004)

$\mathrm{P}(\Theta \mid\{$ data $\}) \propto \mathrm{P}(\{$ data $\} \mid \Theta) \mathrm{P}(\Theta)$

$P(\Theta)$ is the probability of $\Theta$ before taking any account of the new data $\{$ data $\}$, the so-called prior probability.

$P(\{$ data $\} \mid \Theta)$ is the probability of the data assuming that the age $\Theta$ is exact. It is referred as the likelihood and generally denoted by 1 ( $\{$ data $\}$ ).

$P(\Theta \mid\{$ data $\})$ is the probability of $\Theta$ knowing the data $\{$ data\}, the so-called posterior probability. The Bayes' theorem states that the posterior probability is proportional to the product of the prior probability and of the likelihood. Since it is a probability, it is normalized to one.

\section{Models and implementation}

The Bayesian approach requires building models (Buck et al., 1996). Generally, there are a set of models, each successive model introduces a simplification of the real world and is part of a cycle of modelling and tests (Orton, 1980; Box, 1980; Buck et al., 1996). While the proposed schemes in archaeology (Orton, 1980; Buck et al., 1996) generally refer to archaeological and mathematical models, we can add a physical model that includes the choice of the method of analysis, the principle of this method and the sampling (Lanos, 2004).

So we can rewrite Eq. 2.3 as follows:

$$
\mathrm{P}(\boldsymbol{\Theta} \mid\{\text { data }\}) \propto \mathrm{P}(\{\mathbf{d a t a}\} \mid \mathbf{g}, \mathbf{S}, \boldsymbol{\Theta}) \mathrm{P}(\mathbf{g} \mid \mathbf{S}, \boldsymbol{\Theta}) \mathrm{P}(\mathbf{S} \mid \boldsymbol{\Theta})(\mathrm{P}(\boldsymbol{\Theta})
$$

where $\boldsymbol{\Theta}$ denotes the set of dates of interest, $\mathbf{S}$ the archaeological constraints, $\mathbf{g}$ is the set of parameters of the physical model and \{data\} represents the measurements. This equation is similar to the equation posed by Millard (2006a, p.360) according to Lanos (2004) but by explaining the term related to the physical model, as it was published by Lanos (2004, eq. 3.6) in the specific case of archaeomagnetism. We just generalized the meaning of $\mathrm{S}$ to the whole archaeological model and not just the stratigraphic information, e.g. probability of failure (Orton, 1980) or manufacturing dates (Van Strydonck et al., 2004).

Simplifications are not unique. They depend on the knowledge, personal interpretation of the physical world, background experience of the scientific, and among others, the choice of the level of simplification. The cognitive schema creating the model does not fit within the logic of science (Popper, 1982). It can therefore be seen as subjective (Gelman and Shalizi, 2012; Zink and Porto, submitted). By cons, its treatment through the Bayes theorem in the light of the observations should lead to a result (posterior) testable by statistical or graphics methods (Gelman and Shalizi, 2012; Box, 1980).

Thus all the models proposed for the Bayesian treatment of luminescence is relevant, whether it be taking into account only the age and the archaeological model (Rhodes et al., 2003), or that of Huntriss (2008), putting the physical model as a simple ratio between two quantities, provided 'the results of the analysis cannot be better than its assumptions' (Millard, 2006a). However, we agree with Millard (2006a) about the fact that we should try as much as possible to understand the model to limit the risk of correlation between the sources of uncertainties (Aitken, 1985; Zink, 2009). The use of cause-effect diagram must assist us in this task.

The basic model that we propose is based on the cause-effect diagram (Fig. 1). For consistency, we call 'input' the unknown true age and 'output', the observed accumulated dose. Other parameters involved, whether obtained during measurement, or derived from prior knowledge, are considered as influence quantities. The 
choice of the influence quantities depends on the available data which themselves reflect both strategic and opportunistic choices depending on the available facilities and the types of experiments known by the operator.

Thus, in our case, we usually work using the fine grains technique (Zimmerman, 1971; Zink and Porto, 2005), so we use the alpha efficiency. Other choices, such as the quartz inclusions technique, does not involve evaluation of these parameters, but can require, for example, the knowledge of the absorption of the beta radiation (Millard, 2006a; Huntriss, 2008). Moreover, we have chosen to use for the alpha efficiency, the a-value, instead of the k-value (Aitken, 1985) Although, generally, we measure the uranium and thorium contents by alpha counting, in the model we decide to use directly the value of the radionuclide contents, and therefore neglect all calibration parameters and over-counting associated with the alpha counter, included in the overall uncertainty on the contents. Finally, we take into account the correction due to the anomalous fading (Huntley and Lamothe, 2001; Zink, 2008, cf. supra). We could start from a simpler model which does not take account of this correction, and turn into a corrected model if the observed bias was too high.

Our model is not unlike that the one presented by Millard (2006a). It differs only in the influence quantities taken into account. Moreover, Millard (2006a, p. 364) presents an explicit model using several parameters. However, in the body of the article, as well as in the example shown, other influence quantities are mentioned, indicating the suitability of the mathematical model of the physical model data. The biggest difference is probably the introduction in our model of the correction due to fading which is only valid for young ages (linearity range of the growth curve) (Huntley and Lamothe, 2001; Zink, 2008).

Thus, the basic model we propose herein uses the following influence quantities: equivalent dose $D_{e}$, alpha efficiency a, uranium, thorium and potassium contents $\mathrm{C}_{\mathrm{u}}, \mathrm{C}_{\mathrm{h}}, \mathrm{C}_{\mathrm{k}}$, external gamma dose rate $\mathrm{D}_{\gamma}$, cosmic dose rate $D_{c}$, wetness $W_{f}$, wetness parameters $\left\{M_{i}\right\}(i=\{\alpha, \beta\})$, conversion factors $\left\{\mathrm{G}_{\mathrm{ji}}\right\}(\mathrm{j}=\{\mathrm{u}, \mathrm{h}, \mathrm{k}\} ; \mathrm{i}=\{\alpha, \beta\})$, fading factor $\mathrm{g}$, storage time $t_{0}$. The subscripts $\alpha, \beta, \gamma$ refer to the radiations and $\mathrm{u}, \mathrm{h}, \mathrm{k}$ refer to the radionuclide uranium, thorium and potassium.

In the simplest model, the input is the unknown age and the output the equivalent dose (model S.1). We will present also a bit more complex model which corresponds to a single input with multiple outputs such as equivalent dose measured both by TL and OSL, or the measurements of the equivalent doses for several samples of the same object (model S.2). We conclude with a more complex model using multiple inputs and outputs, typically several artefacts from a single level measured by different methods (model S.3).
The evaluation of the posterior can be done by formulating the model in a mathematical form, especially through conjugate prior distributions (Buck et al., 1996). However, the development of software and computational power of the computers now allows a direct implementation of the model through programs. We chose WinBugs software, version for Windows (r) of Bugs (Lunn et al., 2009; Spiegelhalter et al., 1996). This software allows the compilation of the model followed by its numerical implementation using a Markov Chain Monte-Carlo (MCMC) using a Gibbs sampler (Brooks et al., 2011). The MCMC simulation is used to estimate the posterior by sampling a value randomly for each parameter from its probability density function and iterating the process many times. For a sufficiently large number of samplings, the distribution of draws should converge close to the posterior. In the case of the Gibbs sampler, each parameter is sampled successively, and the set of parameters including the new sampled parameter is tested with respect to the model. A didactic presentation of the principle of the MCMCs and the Gibbs sampler can be found in Buck et al. (1996). The parameters used to run the MCMC simulation and to test the convergence is the iterations number within a chain, the number of chains, the number of initial iteration discarded (burn-in period) and the number of iteration between two equally spaced outcomes (thin). The number of iterations must be large enough to reach equilibrium. The burn-in period should discard the initial iterations still influenced by the starting values. Several chains are used to detect the influence of the starting values. Finally, the thinning is designed to save the storage capacity and limit the effect of autocorrelation between successive samplings (Gelman and Shirley, 2011). The values of these parameters are specified with the different models.

\section{CASE STUDIES}

\section{Models}

\section{Case study \#1 - single luminescence date}

Suppose we wish to measure an age by TL and of another by OSL. For the moment, we do not care about the possible relationship between these two ages. We would like to compare our approach with the conventional treatment, i.e. without introducing bias through the choice of the prior on the age, by using a non-informative (or vague) prior (Steel, 2001; Buck et al., 1996). In our case, we assume that the age does not exceed 4000 years.

It is therefore based on the basic model with a single age as input and a single equivalent dose as output (model S.1). For the prior probability on the age, an uniform distribution between present day and 4000 years (abbreviation $\mathrm{U}[0,4 \mathrm{ka}])$ is assumed. 


\section{Case study \#2 - single age using both OSL and TL da- ting}

A common case in luminescence dating is to combine results obtained by different methods (e.g., TL and OSL or Blue-OSL and Infrared-OSL). Assuming that the measurements were made on the same sample, the measured age was thus the same, as well as the radioactivity, including alpha and beta radiation. However, the absorbed dose varied due to the alpha effectiveness. The alpha effectiveness is related to the level of saturation of traps, which is not necessarily identical for different techniques (Zink et al., 2010). The loss of accumulated dose due to anomalous fading is not the same according to the used technique (Zink, 2009) and this causes a further change on the measured archaeological dose.

So the model is a modification of the basic model (case study \#1), using two outputs for one single input; in other words, we use a system of two equation models (Eq. 2.3) with one unknown variable, some model parameters are common to both equations and others are not.

\section{Complex cases}

In practice, the cases may easily become more complex. We may therefore need to date an artefact from multiple samples, themselves dated by various techniques. It may even be that we have to date a phenomenon (archaeological layer, production, cultural period) from several objects of different ages but culturally interconnected. The pinnacle is reached by establishing a chronological sequence.

To handle these cases, it is necessary to establish the hierarchical relationship between the various parameters (Millard, 2006a; Zink, 2009), using the cause-effect relationship between the ages and the measured archaeological equivalent doses. Virtually every case is specific and requires bespoke analysis and computer code (Millard, 2006a).

\section{Case study \#3 - single age from various equivalent doses}

Consider first the case of several samples from the same artefact. In this case, there is no bias between the ages of samples and the age of the object. The age is therefore common to all samples. The calibrations and parameters affect this age in the same way, regardless of the sample. It is the same for the cosmic and gamma radiation. Hence, the age, the calibrations, the parameters, the cosmic and gamma dose rate are common to all samples. On the other hand, alpha and beta dose rate are a priori different because of the inhomogeneity of the clay. Assume further that for one sample, the TL and OSL are measured. The alpha effectiveness and the fading rate depend not only on the mineralogy, but also on the method. They are specific to each method. Fig. 2 shows sche- matically the hierarchical relationships between the parameters.

\section{Case study \#4 - layer dating}

We present only the simple case of a single phenomenon dating from several artefacts. The phenomenon in question may be, for example, an archaeological level (Buck et al., 1996; Millard, 2006a) or the production of a cultural property (Van Strydonck et al., 2004). This model is characterized by artefacts of different ages. The conditions noted in the previous case (case study \#3) concerning the alpha and beta dose rates, the alpha effectiveness and the anomalous fading are the same. If we take the case of an archaeological layer, this also raises the question of size and homogeneity of the layer. Assume the dimensions of the layer of $30 \mathrm{~cm}$, the distances between the artefacts as well as and their dimensions are small compared to $30 \mathrm{~cm}$. Under these conditions, the cosmic and gamma dose rates can be considered homogeneous and therefore independent from the artefacts.

The dating of the layer is characterized by two parameters $\alpha$ and $\beta$ defining the time start and end of the layer (Buck et al., 1996). As in previous cases, they are limited by the time bounds TU and TL.

\section{Data}

In the following examples, we decided to work on simulated cases (real cases anonymized).

Three data sets (called TL1, TL2, OSL1) were used for the luminescence measurements, i.e. the archaeological equivalent dose $D_{\mathrm{e}}$, the alpha effectiveness $a$ and the fading factor $g$ (Table 1). For the dosimetry measurements, we used two data set for internal dose rate, i.e. the radionuclide contents of the ceramic $C_{\mathrm{u}}, C_{\mathrm{h}}, C_{\mathrm{k}}$ (Table 1). The first dose rate set is associated with the luminescence data sets OSL1 and TL1, and the second, to the TL2 set. Only one data set is used for external dose rate, including $\mathrm{D}_{\gamma}, \mathrm{D}_{\mathrm{c}}$ and wetness $\mathrm{W}_{\mathrm{f}}$ (Table 1). Two data sets were used for the time span between irradiation and measurement $t_{0}$, one corresponding to long storage (TL additive

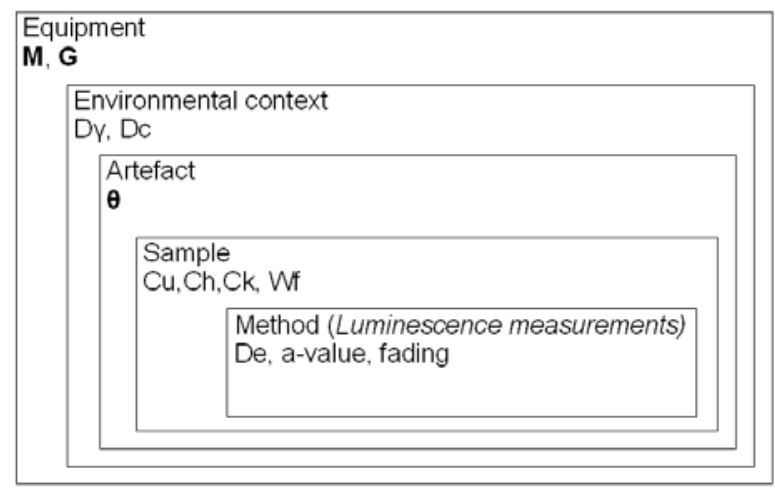

Fig. 2. Hierarchy of the parameters used in case \#3. 
Table 1. Simulated experimental parameters.

\begin{tabular}{|c|c|c|c|c|c|c|c|c|c|c|}
\hline ref & $\mathrm{De}(\mathrm{Gy})$ & a & $g(\% / d)$ & $\mathrm{C}_{\mathrm{u}}(\mathrm{ppm})$ & $\mathrm{C}_{\mathrm{h}}(\mathrm{ppm})$ & $\mathrm{C}_{\mathrm{k}}(\%)$ & $W_{f}(\%)$ & $D_{g}(G y / k a)$ & $D_{c}(G y / k a)$ & $t_{0}(\min )$ \\
\hline TL1 & $14.1 \pm 1.4$ & $0.162 \pm 0.005$ & $0.4 \pm 0.5$ & $2.8 \pm 0.3$ & $8.8 \pm 0.8$ & $3.73 \pm 0.33$ & $5.00 \pm 2.00$ & $0.90 \pm 0.04$ & $0.15 \pm 0.05$ & $31680 \pm 720$ \\
\hline OSL & $11.2 \pm 0.1$ & $0.065 \pm 0.002$ & $0.0 \pm 0.5$ & & & & $5.00 \pm 2.00$ & $0.90 \pm 0.04$ & $0.15 \pm 0.05$ & $2.5 \pm 0.2$ \\
\hline TL2 & $12.1 \pm 1.3$ & $0.077 \pm 0.005$ & $0.2 \pm 0.3$ & $3.1 \pm 0.3$ & $9.8 \pm 0.9$ & $3.29 \pm 0.27$ & $5.00 \pm 2.00$ & $0.90 \pm 0.04$ & $0.15 \pm 0.05$ & $31680 \pm 720$ \\
\hline
\end{tabular}

protocol) and the second to prompt storage (SAR protocol) (Table 1).

The thermoluminescence sets, TL1 and TL2, have large uncertainties on the $D_{\mathrm{e}}(10 \%)$. Such a value is typical of uncertainties obtained by extrapolation methods as additive TL. The OSL1 set has a small uncertainty on the $D_{\mathrm{e}}(1 \%)$ corresponding to an interpolation method, for example, through a single aliquot regeneration protocol (Aitken, 1998).

Some quantities of influence come from the literature. In this paper, these were the cosmic-rays dose rate $D_{\mathrm{c}}$ (Prescott and Hutton, 1994), the wetness parameters $M$ (Zimmerman, 1971), and the conversion factors $\boldsymbol{G}$ (Adamiec and Aitken, 1998). We associated an uncertainty of $5 \%$ but the cosmic dose rate $D_{\text {c }}$ associated with a $33 \%$ uncertainty.

We consider two supplementary temporal constraints: $\mathrm{TL}$ and TU, respectively, terminus ante quem and terminus post quem. As these are ages, not calendar dates, TL and TU are positive or zero, and TL is lower or equal to TU. In our case studies, we will take $\mathrm{TL}=0$ and $\mathrm{TU}=4 \mathrm{ka}$
We will test the cases \#1 and \#2 on the data set TL1 and OSL1. We test the cases \#3 and \#4 on the luminescence set TL1, TL2 and OSL1. We consider in the cases \#2, \#3 and \#4 that TL1 and OSL1 are on the same sample. And in case \#3, all data refer to the same artefact.

\section{Implementation}

Tables 3 to 6 present the formal models, the data and the implementation parameters.

The Bayesian treatment for the first case take $10 \mathrm{sec}-$ onds of computing time for three runs (or chains) of 50,000 iterations, taking every 5 th iteration $($ thin $=5$ ) and discarded the first half (burn-in $=25000$ iterations). The computation time is only slightly higher for the second case ( 15 seconds). It reaches 40 seconds for the third and fourth cases.

Time series for the two tests of the first case are plotted (Fig. 3) taking all iterations without discarded any iteration (no burn-in). It shows fast convergence of age simulations from three chains of 50,000 iterations to an equilibrium level (Figs. 3a and 3b). The three chains are merged, providing proof of the very small influence of
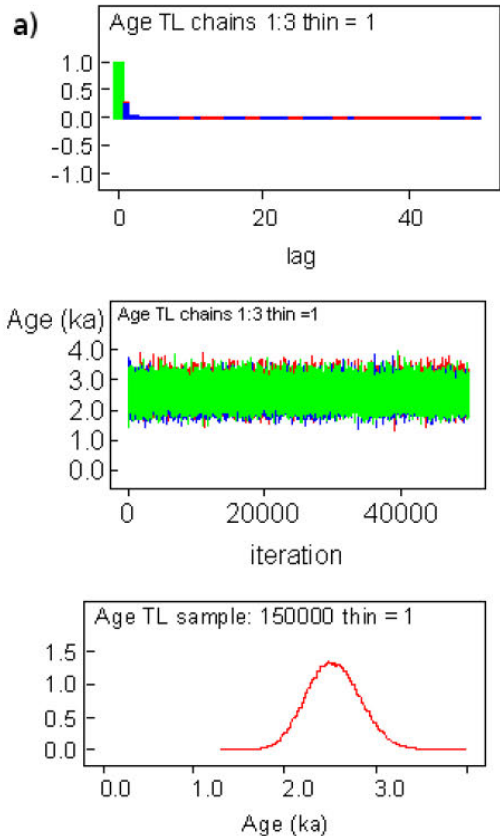

b)
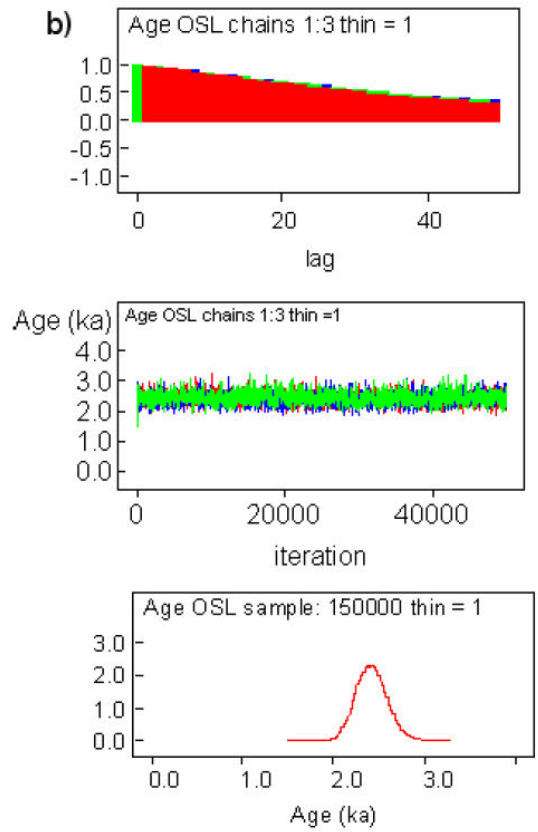
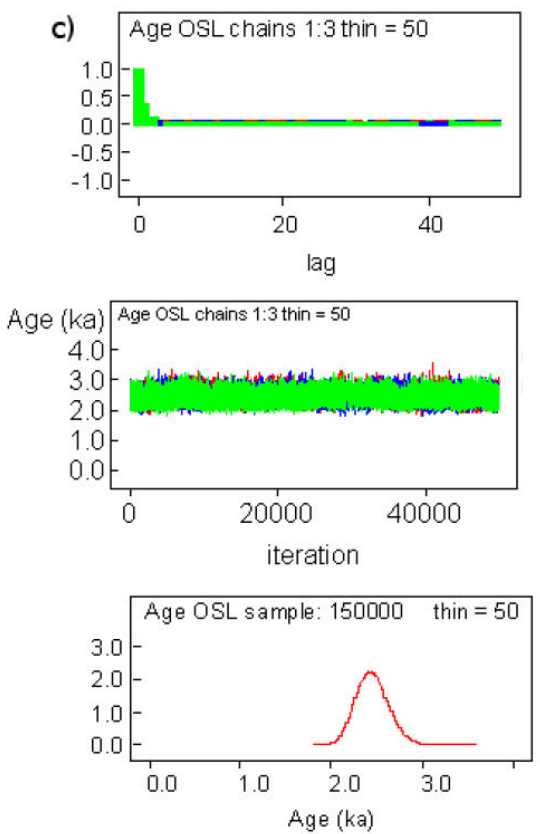

Fig. 3. Analysis of Monte-Carlo Markov Chain simulations (a) data set TL1, 50,000 iterations, 3 chains, thin $=1$ (b) data set OSL, 50,000 iterations, 3 chains, thin $=1$ (c) data set OSL, 2,500,000 iterations, 3 chains, thin $=50$. Top diagrams are the corresponding auto-correlation histograms out to lag 50. Middle diagrams are the time series plots. Bottom diagrams are the posterior distribution of age. 
Table 2. List of parameters of physical model.

\begin{tabular}{lccccc}
\hline & $\begin{array}{c}\text { Wetness parameters } \\
\text { (Zimmerman, 1971) }\end{array}$ & \multicolumn{3}{c}{ Conversion factors (Adamiec and Aitken, 1998) } \\
\hline $\mathrm{Mi}$ & & Gij & Uranium & Thorium & Potassium \\
alpha & $1.50 \pm 0.075$ & alpha & $2.14 \pm 0.107$ & $0.6180 \pm 0.0309$ & - \\
beta & $1.25 \pm 0.0625$ & beta & $0.146 \pm 0.0073$ & $0.0273 \pm 0.001365$ & $0.6490 \pm 0.03245$ \\
\hline
\end{tabular}

Table 3. Bayesian model - single age Case \#1.

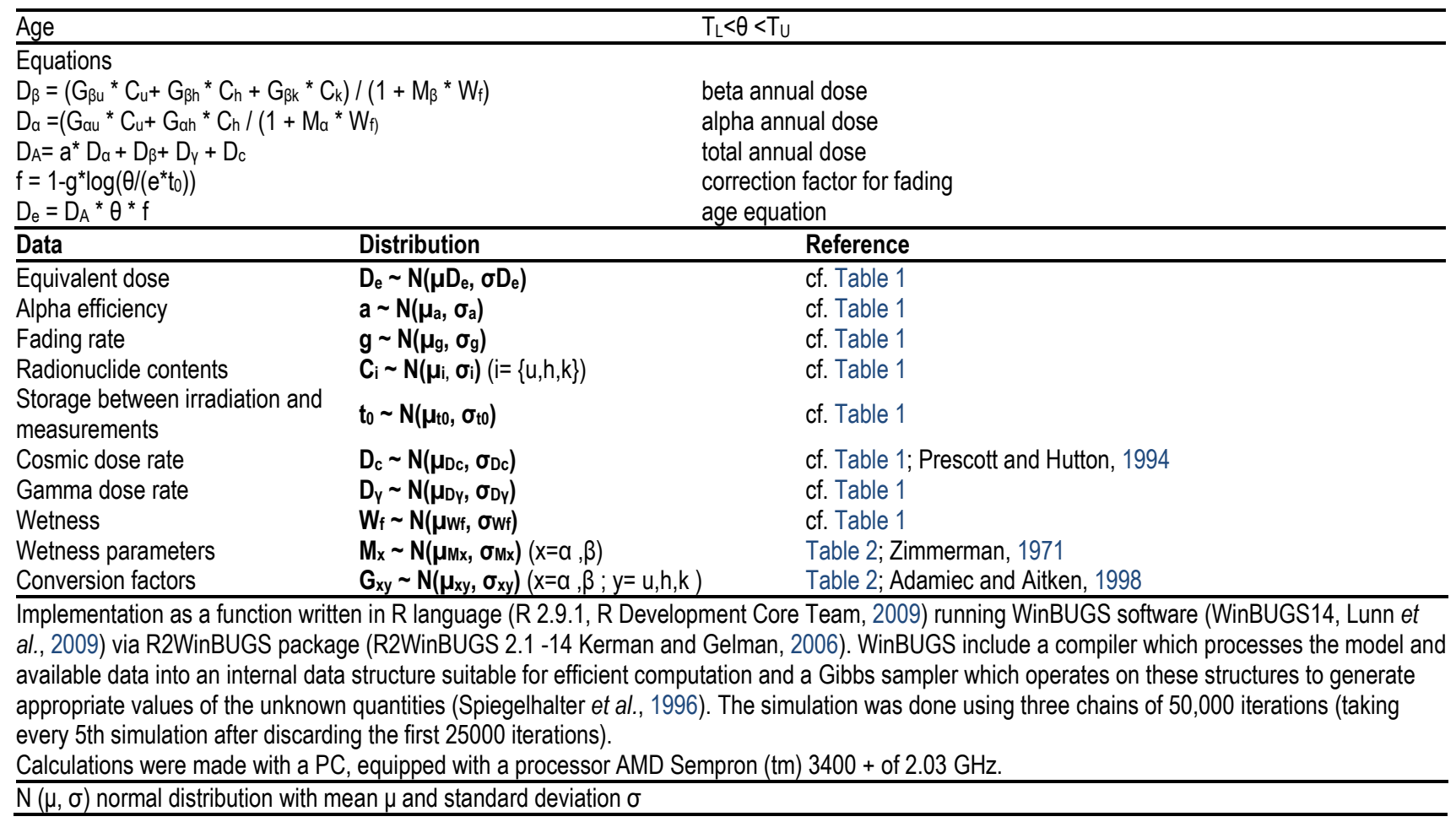

Table 4. Bayesian model - Case \#2

\begin{tabular}{ll}
\hline Age & TL $<\theta<T u$ \\
\hline Equations & \\
$D_{\beta}=\left(G_{\beta u}{ }^{*} C_{u}+G_{\beta h}{ }^{*} C_{h}+G_{\beta k}{ }^{*} C_{k}\right) /\left(1+M_{\beta}{ }^{*} W_{f}\right)$ & beta annual dose \\
$D_{a}=\left(G_{a u}{ }^{*} C_{u}+G_{a h}{ }^{*} C_{h} /\left(1+M_{a}{ }^{*} W_{f}\right)\right.$ & alpha annual dose \\
$D_{A i}=a_{i}^{*} D_{a}+D_{\beta}+D_{y}+D_{c}$ & total annual dose for method $i$ \\
$f_{i}=1-g_{i}^{*} \log \left(\theta /\left(e^{*} t_{0 i}\right)\right)$ & correction factor for fading for method $i$ \\
$D_{e i}=D_{A i}{ }^{*} \theta * f_{i}$ & age equation for method i \\
\hline
\end{tabular}

Same parameters as for Table 3.

Table 5. Bayesian model - Case \#3

\begin{tabular}{|c|c|}
\hline Age & $\mathrm{T}_{\mathrm{L}}<\theta<\mathrm{T}_{\mathrm{U}}$ \\
\hline Equations & \\
\hline$D_{\beta i}=\left(G_{\beta u}{ }^{*} C_{u i}+G_{\beta h}{ }^{*} C_{h i}+G_{\beta k}{ }^{*} C_{k i}\right) /\left(1+M_{\beta}{ }^{*} W_{f i}\right)$ & beta annual dose for sample i \\
\hline$D_{a i}=\left(G_{a u}{ }^{*} C_{u i}+G_{a h}{ }^{*} C_{h i} /\left(1+M_{a}{ }^{*} W_{f i}\right)\right.$ & alpha annual dose for sample i \\
\hline$D_{A i}=a^{*} D_{a i}+D_{\beta i}+D_{\gamma}+D_{c}$ & total annual dose for sample i \\
\hline$f_{i}=1-g_{i}^{*} \log \left(\theta /\left(e^{*} t_{0 i}\right)\right)$ & correction factor for fading for sample i \\
\hline$D_{e i}=D_{A i} * \theta * f_{i}$ & age equation for sample i \\
\hline
\end{tabular}

Same parameters as for Table 3. 


\begin{tabular}{|c|c|}
\hline Age & $T_{L}<\alpha<\theta i<\beta<T_{U}$ \\
\hline Equations & \\
\hline$D_{\beta i}=\left(G_{\beta u}{ }^{*} C_{u i}+G_{\beta h}{ }^{*} C_{h i}+G_{\beta k}{ }^{*} C_{k i}\right) /\left(1+M_{\beta}{ }^{*} W_{f i}\right)$ & beta annual dose for sample i \\
\hline$D_{a i}=\left(G_{a u}{ }^{*} C_{u i}+G_{a h}{ }^{*} C_{h i} /\left(1+M_{a}{ }^{*} W_{f i}\right)\right.$ & alpha annual dose for sample i \\
\hline$D_{A i j}=a_{i j}^{*} D_{a i}+D_{\beta i}+D_{Y}+D_{c}$ & total annual dose for sample i and method j \\
\hline$f_{i j}=1-g_{i j}^{*} \log \left(\theta /\left(e^{*} t_{0 i j}\right)\right)$ & correction factor for fading for sample $\mathrm{i}$ and method $\mathrm{j}$ \\
\hline$D_{\text {eij }}=D_{\text {Aij }}{ }^{*} \theta * f_{i j}$ & age equation for sample $\mathrm{i}$ and method $\mathrm{j}$ \\
\hline
\end{tabular}

Same parameters as for Table 3.

the starting values. The auto-correlation for TL 1 data set shows that the correlation was limited to a short range (lag $<3$; Fig. 3a), while for the OSL ( $D_{\mathrm{e}}$ known with best precision), auto correlation was present up to lag 50 (Fig. 3b). Taking every 50th iteration (or a thinning of 50), the auto correlation for OSL was reduced to short range (Fig. 3c). The new result obtained for thin $=50(2,500,000$ iterations and a burn-in of 1,250,000, 700 second of computing time) was in agreement with that obtained for thin $=5$.

In the case \#2, the characteristics of the MCMC simulations, especially auto-correlation, are similar to the measurement of OSL in the case \#1, since it is the most accurate measurement.

\section{INTERPRETATION}

The age obtained using the classical approach are presented in Table 7. Table 8 postpones the balance of uncertainties in the case of the TL obtained by classical approach, (Aitken, 1985; Zink, 2009).
Using for the age, a uniform prior $U[0,4 \mathrm{ka}]$, the Bayesian treatment of the case \#1 led to a TL1 age of $2555 \pm 300$ years $( \pm 11.8 \%)$ and an OSL1 age of $2424 \pm 179$ years $( \pm 7.3 \%)$. The agreement between both approaches is good. The difference between the uncertainties is not significant and can be attributed to differences in numerical rounding.

Similar results are obtained for the case \#2. The weighted average age between both methods according to Aitken (1985) is of $2450( \pm 6.6 \%)$. Bayesian treatment from a non-informative prior on the age ( $U$ [0.4 ka]) gives us an age of $2442 \pm 163( \pm 6.7 \%)$.

In case \#3, the conventional analysis based on Aitken (1985) is difficult. What are the sources of systematic and random uncertainties, knowing that TL1 and OSL1 are made on the same sample then some of their uncertainties have common origin, therefore can be regarded as systematic, but are not shared with TL2 corresponding to a second sample? We assess the average age at $2470 \pm 140$ years $(5.7 \%)$, without being quite sure of our analysis.

Table 7. Luminescence analysis - classical approach.

\begin{tabular}{|c|c|c|c|c|c|c|c|c|c|c|c|c|c|}
\hline & $D_{e}(G y)$ & $\underset{(p p m)}{U}$ & $\begin{array}{c}\text { Th } \\
\text { (ppm) }\end{array}$ & $\mathrm{K}_{2} \mathrm{O}(\%)$ & $\begin{array}{l}\text { Effectiveness } \\
\text { alpha }\end{array}$ & $\begin{array}{c}D_{\alpha}^{\prime} \\
\text { (mGy/a) }\end{array}$ & $\begin{array}{c}D_{\beta} \\
\text { (mGy/a) }\end{array}$ & $\begin{array}{c}D_{\text {ext }} \\
\text { (mGy/a) }\end{array}$ & $\begin{array}{c}\text { DA } \\
\text { (mGy/a) }\end{array}$ & $\begin{array}{l}\text { Obse- } \\
\text { rved } \\
\text { Age } \\
\text { (years) }\end{array}$ & $\begin{array}{l}\text { Fading } \\
\text { rate } \\
\text { (\%/decade) }\end{array}$ & $\begin{array}{c}\text { Age } \\
\text { (years) }\end{array}$ & $\begin{array}{c}\text { Unce- } \\
\text { rtainties } \\
(\%)\end{array}$ \\
\hline TL1 & $14.1 \pm 1.4$ & $2.8 \pm 0.3$ & $8.8 \pm 0.8$ & $3.73 \pm 0.33$ & $0.162 \pm 0.005$ & 1.72 & 2.89 & $1.05 \pm 0.05$ & 5.66 & 2490 & $0.4 \pm 0.5$ & 2535 & \pm 11.8 \\
\hline OSL1 & $11.2 \pm 0.1$ & $2.8 \pm 0.3$ & $8.8 \pm 0.8$ & $3.73 \pm 0.33$ & $0.065 \pm 0.002$ & 0.69 & 2.89 & $1.05 \pm 0.05$ & 4,63 & 2420 & $0.0 \pm 0.5$ & 2420 & \pm 6.8 \\
\hline TL2 & $12.1 \pm 1.3$ & $3.1 \pm 0.3$ & $9.8 \pm 0.9$ & $3.29 \pm 0.27$ & $0.077 \pm 0.005$ & 0.91 & 2.69 & $1.05 \pm 0.05$ & 4.65 & 2600 & $0.2 \pm 0.3$ & 2620 & \pm 12.5 \\
\hline
\end{tabular}

De - Equivalent dose to the archaeological dose received by the sample since its last heating - unit Gray - Gy

$U, T h, \mathrm{~K}_{2} \mathrm{O}$ radionuclides contents

Alpha effectiveness - correction factor reflecting alpha dose rate in equivalent beta (or gamma) dose rate - It is the a-value as defined in Aitken (1985) - dimensionless unit 1

$D_{\alpha}^{\prime}-$ contribution of alpha particles to the annual dose: $D_{\alpha}^{\prime}=a \times(2.31 \times U+0.611 \times$ Th) $/(1+1.5 \times$ WF) (Adamiec and Aitken, 1998) - unit milligray per year - $m G y / a$

$D_{\beta}$ - contribution of beta radiation to the annual dose rate: $D_{\beta}=(0.146 \times U+0.0273 \times T h+0.649 \times K 20) /(1+1.25 \times$ WF $)$ (Adamiec and Aitken, 1998) - unit milligray per year - $m$ Gy/a

(WF- Wetness $5 \pm 2 \%$ )

$D_{\text {ext }}$ - contribution of the environment (cosmic and gamma) to the annual dose rate - Gamma dose rate 0.90 ( $\pm 5 \%$ ) and cosmic rays dose rate 0.15 ( $\pm 33 \%)$ based on tables provided by Prescott and Hutton, 1994.

$D A$ - annual dose or dose rate - dose received by the sample during one year - it is composed by contributions in alpha, beta, gamma and cosmic Unit milligray per year - $m G y$ / a (equivalent to Gray per kilo-annum (thousand years) - Gy / ka)

Observed age - age determined from the equation of age regardless of the fading correction of observed age $=D_{e} / D A-$ unit year.

Fading rate - correction factor due to anomalous fading - unit percent per decade -\% / decade.

Age - ages determined after fading correction (Zink, 2008).

Uncertainty - based on the combined standard uncertainties as described in Table 8. 
Table 8. Summary of standard uncertainty components.

\begin{tabular}{|c|c|c|c|c|}
\hline Source of uncertainty & Type & $U(x i)$ & $\mathrm{Ci}$ & Uri(y) \\
\hline Luminescence measurements Ur1 & $A$ & & & $10.0 \%$ \\
\hline Archaeological dose UrQ (TL) & A & $10.0 \%$ & 1.0 & $10.0 \%$ \\
\hline Alpha Effectiveness Ury (TL) & A & $3.0 \%$ & 0.30 & $0.90 \%$ \\
\hline Annual dose measurements Ur2 & A & & & $4.67 \%$ \\
\hline X-rays analysis UU & A & $10.0 \%$ & 0.23 & $2.30 \%$ \\
\hline X-rays analysis UTh & A & $10.0 \%$ & 0.18 & $1.80 \%$ \\
\hline X-rays analysis UK & A & $8.8 \%$ & 0.40 & $3.50 \%$ \\
\hline External gamma dose rate & A & $5.0 \%$ & 0.16 & $0.80 \%$ \\
\hline External cosmic dose rate & A & $20.0 \%$ & 0.03 & $0.60 \%$ \\
\hline Calibrations Ur4 & $A / B$ & & & $3.81 \%$ \\
\hline Beta source & $\mathrm{B}$ & $5.0 \%$ & 0.70 & $3.50 \%$ \\
\hline Alpha Source & B & $5.0 \%$ & 0.30 & $1.50 \%$ \\
\hline Parameters Ur5 & $B$ & & & $2.96 \%$ \\
\hline Parameters linked to alpha & B & $5.0 \%$ & 0.30 & $1.50 \%$ \\
\hline Parameters linked to beta & B & $5.0 \%$ & 0.51 & $2.55 \%$ \\
\hline Wetness Ur7 & $B$ & $25.0 \%$ & 0.06 & $1.50 \%$ \\
\hline Fading Ur10 (TL) & $\mathrm{A}$ & & & $2.94 \%$ \\
\hline Combined standard uncertainty Urc & & & & $12.5 \%$ \\
\hline
\end{tabular}

The values presented here are based on the average values of the various samples.

Type: A - uncertainty evaluated by statistical analysis; $B$ - uncertainty evaluated by means other than statistical analysis.

$U(x i)$ estimated uncertainty of the input $x i$

$\mathrm{Ci}$ sensitivity coefficient $\mathrm{Ci}=\partial \mathrm{y} / \partial \mathrm{xi}$

Uri(y) contribution due to the input $x$ in the total uncertainty Uri(y) $=\mathrm{Ci} U(x i)$

The calculation of uncertainties UR1 to Ur7 is based the appendix B of Aitken (1985).

The fading, Ur10 is estimated by comparing the standard deviations of Monte-Carlo simulation with and without fading (Zink, 2008).

The combined standard uncertainty Urc is the square root of the quadratic sum of standard uncertainties: Urc ${ }^{\wedge} 2=\Sigma U_{r i}^{\wedge} 2(I=1,2,4,5,7,10)$.

Bayesian treatment gives an age of $2530 \pm 165$ years (6.5\%). Analysis using the model of Huntriss (2008) gives a result similar to the conventional method $2490 \pm 140$ years. This seems to indicate that the uncertainty estimate by the conventional method is too optimistic.

The shift to higher ages given by our method compared to the result of the conventional method is explained by the uncertainty of fading in OSL. Because the storage time after irradiation is very short in SAR-OSL, the uncertainty contribution due to the anomalous fading on the age is higher in OSL (Zink, 2008). Moreover, in this case, the uncertainty on the measurement of the fading rate is high which increases the uncertainty. Using an uncertainty on the fading rate of $0.25 \%$ / decade instead of $0.5 \%$ / decade, the age obtained by our method, $2470 \pm 145$ years, is consistent with the conventional method. We assume that the conventional method underestimates the error introduced by the anomalous fading in this slightly complex case.

In case \#4, we obtain for the first object (TL1/OSL1) an age of $2505 \pm 170$ years $( \pm 6.8 \%)$ and for the second object of $2585 \pm 255$ years $( \pm 9.9 \%)$. The age gap is lower than if we had not introduced the lower and upper bounds for the archaeological level. We used as prior for each age a constant density between the two bounds (Buck et al., 1996). Our sampling number is too low for a correc- tion of the prior as proposed by Nicholls and Jones (2001) will be useful.

Our model follows the rules of Bayesian treatment. In the limiting case of a vague prior, we find the results given by the classical treatment. Similarly, if the prior uncertainty is very weak, the posterior is identical to the prior, i.e. the luminescence measurements provided no additional information. Moreover, the simulation shows a quick convergence. Our model seems correct.

\section{DISCUSSION AND CONCLUSIONS}

Although our model is relatively simple, there is a good fit between the standard approach and the Bayesian approach in the case of non-very informative prior. The result is independent of the MCMC simulations, as shown by the good overlap between the different chains. The chains converge quickly. We discarded, however, systematically the first half, i.e. the first 25,000 iterations, as a precaution. The auto-correlation strongly depends on the uncertainty of the data. It is limited to short distance in the case where uncertainty is large, corresponding to $10 \%$ on the equivalent dose, but can affect long-range (50 lag) where the uncertainty falls to $1 \%$ on the equivalent dose. In this case, it is necessary to take only one iteration every fifty iterations. It increases the number of 
iterations necessary to achieve the same final number of effective iterations. However, the results are consistent.

The Bayesian approach proposed, although relatively simple, gives satisfactory results.

Using a Bayesian compiler, in the present case WinBugs, allows for non-statistician to overcome the analytical development of the equations while enjoying a relatively fast computation time ( 15 seconds for a single dating on a standard desktop computer).

The approach we followed is similar to that proposed by Millard (2004, 2006a, 2006b). Beyond the single archaeological model that included stratigraphic relationships, the lifetimes of artefact, etc., we consider the experimental or physical model. It takes into account the parameters of the study, measured or obtained in another way, and their hierarchical relationship. There is therefore no universal model, but models not only adapted to the case studied, but also to the experimental approach depending, amongst other things, on the knowledge and background of the physicist and of the available technology. Hence, Millard (2006a) presents a model, but in the example of the article, he uses other models parameters like alpha effectiveness and beta absorption. Our model is characterized, compared to Millard's, by taking into account the correction for the effect fading in the case of samples of recent age.

I have presented here a small number of variations from our basic model. We can made the model more complex, as is the case in an article under submission (Zink et al., submitted) that takes into account additional parameters such as dose rate of the laboratory sources. We can also, for example, introduce in the case \#2 a bias between TL and OSL ages from the same sample. But, the introduction of such a bias must correspond to an initial hypothesis which allows us to specify the prior of this bias. It is needed if we want to test the hypothesis. The systematic introduction of bias without bespoke hypothesis leads to quantification of the bias through its posterior without knowing the reality of the bias (risk of false positive). Always focus on initial models with reduced numbers of parameters (Occam's razor, Zink and Porto, submitted). Our system is limited to the passage between the equivalent dose and age. It must be borne in mind that a number of uncertainties are not taken into account, as the source calibration uncertainties which are shared between samples. Hence the age results are not fully independent. The following work aims to extend our model so that it uses more data. Obviously the more we data is introduced, the more the calculation time will lengthen. And it is not certain whether a global Bayesian treatment of luminescence dating may be possible.

\section{REFERENCES}

Adamiec G and Aitken MJ, 1998. Dose-rate conversion factors: update. Ancient TL 16: 37-50.

Aitken MJ, 1985. Thermoluminescence dating, Studies in Archaeological Science. Academic press, London.
Aitken MJ, 1998. An introduction to Optical dating - The dating of quaternary sediments by the use of photon-stimulated luminescence. Oxford Science Publication. Oxford University Press, Oxford.

Box GEP, 1980. Sampling and Bayes' Inference in Scientific Modelling and Robustness. Journal of the Royal Statistical Society. Series A (General) 143: 383.

Brooks S, Gelman A, Jones GL and Meng X-L (Eds.), 2011. Handbook of Markov Chain Monte Carlo - CRC Press Book. Chapman \& Hall/CRC Handbooks of Modern Statistical Methods. CRC Press.

Buck CE and Millard AR (Eds.), 2004. Tools for Constructing Chronologies, 1 st ed, Lecture Note in Statistics. Springer, London.

Buck CE, Cavanagh WG and Litton CD, 1996. Bayesian approach to interpreting archaeological data, Statistics in Practice. John Wiley \& Sons, Inc., Chichester.

Clark-Balzan LA, Candy I, Schwenninger J-L, Bouzouggar A, Blockley S, Nathan R and Barton RNE, 2012. Coupled U-series and OSL dating of a Late Pleistocene cave sediment sequence, Morocco, North Africa: Significance for constructing Palaeolithic chronologies. Quaternary Geochronology 12: 53-64, DOI 10.1016/j.quageo.2012.06.006.

Cox RT, 1946. Probability, Frequency, and Reasonable Expectation. American Journal of Physics 14: 1-13, DOI 10.1119/1.1990764.

Gelman A and Shalizi CR, 2012. Philosophy and the practice of Bayesian statistics. British Journal of Mathematical and Statistical Psychology.

Gelman A and Shirley K, 2011. Inference from simulations and monitoring convergence, in: Brooks S, Gelman A, Jones GL and Meng X-L (Eds.), Handbook of Markov Chain Monte Carlo - CRC Press Book, Chapman \& Hall/CRC Handbooks of Modern Statistical Methods. CRC Press, pp. 163-174.

Huntley DJ and Lamothe M, 2001. Ubiquity of anomalous fading in Kfeldspars and the measurement and correction for it in optical dating. Canadian Journal of Earth Sciences 38(7): 1093-1106, DOI 10.1139/e01-013.

Huntriss A, 2008. A Bayesian analysis of luminescence dating. PhD, University of Durham.

Kerman J and Gelman A, 2006. Bayesian Data Analysis using R. Rnews 6: $21-24$.

Lanos P, 2004. Bayesian inference of calibration curves: Application to Archaeomagnetism, in: Buck CE and Millard AR (Eds.), Tools for Constructing Chronologies, Lecture Note in Statistics. Springer, London, pp. 43-82.

Lunn D, Spiegelhalter D, Thomas A and Best N, 2009. The BUGS project: Evolution, critique and future directions. Statistics in Medicine 28(25): 3049-3067, DOI 10.1002/sim.3680.

Millard AR, 2004. Taking Bayes beyond radiocarbon: Bayesian approaches to some other chronometric methods, in: Buck, CE, Millard AR (Eds.), Tools for Constructing Chronologies, Lecture Note in Statistics. Springer, London, pp. 231-248.

Millard AR, 2006a. Bayesian analysis of pleistocene chronometric methods. Archaeometry 48(2): 359-375, DOI 10.1111/j.14754754.2006.00261.x.

Millard AR, 2006b. Bayesian analysis of ESR dates, with application to Border Cave. Quaternary Geochronology 1(2): 159-166, DOI 10.1016/j.quageo.2006.03.002.

Murray AS and Wintle AG, 2003. The single aliquot regenerative dose protocol: potential for improvements in reliability. Radiation Measurements 37(4-5): 377-381, DOI 10.1016/S13504487(03)00053-2.

Nicholls G and Jones M, 2001. Radiocarbon dating with temporal order constraints. Journal of the Royal Statistical Society: Series C (Applied Statistics) 50(4): 503-521, DOI 10.1111/1467-9876.00250.

Orton C, 1980. Mathematics in Archaeology. 1st ed, Collins archaeolgy. Wiliams Collins Sons \& Co, London.

Popper K, 1982. La logique de la découverte scientifique (The logic of scientific discovery). 2nd French ed. Payot, Paris (in French).

Prescott JR and Hutton JT, 1994. Cosmic ray contributions to dose rates for luminescence and ESR dating: large depths and long-term time variations. Radiation Measurements 23(2-3): 497-500, DOI 10.1016/1350-4487(94)90086-8,. 
Preusser F, Degering D, Fuchs M, Hilgers A, Kadereit A, Klasen N, Krbetschek M, Richter D and Spencer JQG, 2008. Luminescence dating: basics, methods and applications. Quaternary Science Journal 57(1-2): 95-149, DOI 10.3285/eg.57.1-2.5.

$\mathrm{R}$ Development Core Team, 2009. $R$ : A language and environment for statistical computing. R Foundation for Statistical Computing, Vienna, Austria. ISBN 3-900051-07-0, WEB site: <http://www.Rproject.org>. Accessed 2010 April 14.

Rhodes EJ, Bronk Ramsey C, Outram Z, Batt C, Willis L, Dockrill S and Bond J, 2003. Bayesian methods applied to the interpretation of multiple OSL dates: high precision sediment ages from Old Scatness Broch excavations, Shetland Isles. Quaternary Science Reviews 22(10-13): 1231-1244, DOI $10.1016 / \mathrm{S} 0277-$ 3791(03)00046-5.

Sivia DS, Burbidge C, Roberts RG and Bailey RM, 2004. A Bayesian approach to the evaluation of equivalent doses in sediment mixtures for luminescence dating, in: AIP Conference Proceedings. p. 305.

Sommer KD and Siebert BRL, 2006. Systematic approach to the modelling of measurements for uncertainty evaluation. Metrologia 43(4): S200-S210, DOI 10.1088/0026-1394/43/4/S06.

Spiegelhalter D, Thomas A, Best N and Gilks W, 1996. BUGS 0.5: Bayesian inference using Gibbs sampling manual (version ii). MRC Biostatistics Unit, Institute of Public Health, Cambridge, UK.

Steel D, 2001. Bayesian statistics in radiocarbon calibration. Philisophy of Sciences 68(3): S153-S164.

Van Strydonck M, Moor AD and Benazeth D, 2004. 14C dating compared to art historical dating of roman and coptic textiles from egypt. Radiocarbon 46: 231-244.

Wintle AG, 1973. Anomalous Fading of Thermo-luminescence in Mineral Samples. Nature 245: 143-144, DOI 10.1038/245143a0.
Wintle AG, 2008. Luminescence dating of Quaternary sediments Introduction. Boreas 37(4): 469-470, DOI 10.1111/j.15023885.2008.00060.x.

Zimmerman DW, 1971. Thermoluminescent Dating Using Fine Grains from Pottery. Archaeometry 13(1), 29-52, DOI 10.1111/j.14754754.1971.tb00028.x.

Zink A, 2008. Uncertainties on the Luminescence Ages and Anomalous Fading. Geochronometria 32: 47-50, DOI 10.2478/v10003-008$0027-4$.

Zink A, 2009. Luminescence date and archaeological ages: An epistemology of the luminescence dating. in: Cavulli F, Prudêncio MI, DIAS MI (Eds.), Defining a Methodological Approach to Interpret Structural Evidence; Archaeometry, British Archaeological Report. Presented at the XV UISPP World Congress (Lisbon, 4-9 September 2006) / XV Congrès Mondial (Lisbonne, 4-9 Septembre 2006, Vol 32, Sessions WS28, C69, C70 and C71., Archaeopress, pp. 113-116.

Zink A and Porto E, 2005. Luminescence dating of the Tanagra terracottas of the Louvre collections. Geochronometria 24: 21-26.

Zink AJC, Dabis S, Porto E and Castaing J, 2010. Alpha efficiency under TL and OSL - A subtraction technique using OSL and TL to detect artificial irradiation. Radiation Measurements 45(3-6): 649652, DOI 10.1016/j.radmeas.2010.01.017.

Zink AJC and Porto E, submitted. Bayesian Approach or Robust Bayesian analysis? Quaternary Geochronology.

Zink AJC, Porto E, Genevey A, Gallet Y, Rante R and Collinet A, submitted. Absolute chronology of Nishapur's Citadel (Iran). Contribution of luminescence dating and archaeomagnetic analysis. Quaternary Geochronology.

Zink AJC, Susino GJ, Porto E and Huffman TN, 2012. Direct OSL dating of Iron Age pottery from South Africa - Preliminary dosimetry investigations. Quaternary Geochronology 8: 1-9, DOI 10.1016/j.quageo.2011.11.008. 